\title{
Matrix-Assisted Laser Desorption/Ionization Mass Spectrometric Analysis of Polysulfated-Derived Oligosaccharides Using Pyrenemethylguanidine
}

\author{
Keiichiro Ohara, ${ }^{\mathrm{a}}$ Jean-Claude Jacquinet, ${ }^{\mathrm{b}}$ Diane Jouanneau, ${ }^{\mathrm{c}}$ \\ William Helbert, ${ }^{c}$ Michael Smietana, ${ }^{a}$ and Jean-Jacques Vasseur ${ }^{a}$ \\ ${ }^{a}$ Institut des Biomolécules Max Mousseron, UMR 5247 CNRS, Université Montpellier 1 and Université \\ Montpellier 2, Montpellier, France \\ ${ }^{\mathrm{b}}$ Institut de Chimie Organique et Analytique, UMR6005, CNRS, Université d'Orléans, Orléans, France \\ ${ }^{c}$ Marine Plants and Biomolecules, UMR 7139, CNRS, Université Pierre et Marie Curie, Roscoff, France
}

\begin{abstract}
A better understanding of the biological roles of carbohydrates requires the use of tools able to provide efficient and rapid structural information. Unfortunately, highly acidic oligomerssuch as polysulfated oligosaccharides-are very challenging to characterize because of their high polarity, structural diversity, and sulfate lability. These features pose special problems for matrix-assisted laser desorption/ionization mass spectrometric (MALDI-MS) analysis because polysulfated carbohydrates exhibit poor ionization efficiency and usually do not produce any signal. The present report demonstrates how MALDI-MS can be used to derive structural and compositional information from pure and mixed fractions of polysulfated oligosaccharides. Indeed, pyrenemethylguanidine (pmg, a derivatizing agent and ionization efficiency enhancer) was used for the analysis of di- to decasaccharides, carrying from two to nine sulfate groups. The method is applied to various highly sulfated chondroitin and carrageenan oligosaccharides as well as to the analysis of mixtures of compounds. In the mass spectra, the observation of a unique pmg-complexed ladder of peaks in both ionization modes allows an easy and rapid determination of both the number of sulfate groups carried by the analyte and its molecular weight. Moreover, we have developed a software tool for the rapid and automatic structural elucidation of carrageenans based on the mass spectra obtained. (J Am Soc Mass Spectrom 2009, 20, 131-137) ( 2009 Published by Elsevier Inc. on behalf of American Society for Mass Spectrometry
\end{abstract}

$\mathrm{T}$ The high negative charge, polydispersity, and sequence heterogeneity of complex carbohydrates render their structural elucidation one of the most difficult challenges for chemists, often requiring the application of multiple analytical approaches [1]. Furthermore, polysulfated carbohydrates are important biologically active molecules involved in many physiological and pathophysiological conditions. Glycosaminoglycans (GAGs), for example, are central players in blood coagulation, signal transduction, or cell-cell recognition [2-4]. Carrageenans, another important class of complex carbohydrates, are water-soluble linear sulfated galactans extracted from marine red algae, serving mainly as gelling or stabilizing agents in the food industry [5]. They also have potential as pharmaceuticals $[6,7]$. Interestingly, it has been demonstrated that many polysulfated polysaccharides may have potent antiviral properties, depending on the position and the

Address reprint requests to Dr. Michael Smietana, Université Montpellier 2, Institut des Biomolécules Max Mousseron (IBMM), UMR 5247 CNRS, Place Eugène Bataillon, 34095 Montpellier Cedex 5, France. E-mail: msmietana@ univ-montp2.fr density of the $O$-sulfates, as well as the size of the polysaccharide moiety [7]. In addition, synthetic polysulfated carbohydrate drugs-such as the antiulcer sucrose octasulfate or the octasulfated pentasaccharide anticoagulant Arixtra-are chemically prepared by $O$ sulfonation of oligosaccharide precursors. Considering the myriad of polysulfated carbohydrate structures, their structural diversity, and their potential importance to the food and pharmaceutical industries, strong analytical tools are needed to gain in-depth structural information. Because of their chemical heterogeneity and highly acidic character, polysulfated carbohydrates are challenging analytes. Nuclear magnetic resonance spectroscopy is a powerful technique for the characterization of such oligomers, but large amounts of product are needed and mixtures of compounds are difficult to study [8]. Mass spectrometry is another useful analytical method, particularly because it allows one to deduce structural details from molecular weight information [9]. Unfortunately, the polyanionic nature of these compounds renders it difficult to generate intact gas-phase ions for mass spectrometric analysis because polysul-
(C) 2009 Published by Elsevier Inc. on behalf of American Society for Mass Spectrometry. 1044-0305/09/\$32.00

doi:10.1016/j.jasms.2008.09.004
Published online September 6, 2008 Received July 7, 2008 Revised September 1, 2008 Accepted September 1, 2008 
fated carbohydrates have a tendency to lose $\mathrm{SO}_{3}$ groups ( $\Delta-80$ mass units). Although electrospray ionization (ESI) mass spectrometry is one of the preferred approaches for the analysis of highly acidic compounds, it has been shown that the data interpretation is complicated because of the creation of multiply charged ions [10-14]. Nevertheless, MALDI-MS has not been used for the detection of such compounds, although MALDIMS has the advantage of creating mostly singly charged ions because polysulfated carbohydrates possess a poor ability to ionize. Because of their propensity to form adduct with cations, sulfated oligosaccharides had to be complexed with basic peptides, such as (Gly-Arg) where $n$ exceeds the number of sulfate groups by one, for efficient MALDI-TOF MS analysis [15-17]. Although this complexation method is efficient and sensitive, it is hampered by the requirement of having a library of peptides suitable for the analysis of unknown compounds. Tremendous advances were recently made for MALDI detection of alkali metal salts of sulfated carbohydrates either using ionic liquid matrices [18-20] or by alkali metal exchange [21]. These methods are very efficient since they partially suppress $\mathrm{SO}_{3}$ loss but, unfortunately, they cannot avoid $\mathrm{Na} / \mathrm{H}$ or $\mathrm{Cs} / \mathrm{Na}$ exchange peaks and the mass spectra obtained might be difficult to interpret. Moreover, the analysis and the structural elucidation of unknown compounds remain a difficult task. Therefore, the challenge remains to develop a simple method using a benchtop MALDI-time of flight (TOF) mass spectrometer for the characterization of highly sulfated carbohydrates. Although a large body of work has been devoted to prevent fragmentation of the parent ions of highly sulfated oligosaccharides, we thought that specific fragments resulting from $\mathrm{SO}_{3}$ loss might be very useful in obtaining structural information. The benefit would result from fragmented mass spectra only if (1) specific and reproducible fragmentations are observed and (2) glycosidic fragments or exchange peaks are avoided.

Recently, we used MALDI-TOF mass spectrometry to observe complexes between single-strand oligonucleotides and various structurally distinct guanidinium derivatives. This study revealed the importance of the guanidinium group for an efficient interaction with the phosphodiester linkages of DNA and emphasized the importance of the presence of an aromatic ring on the artificial receptor. In particular, pyrenemethylguanidine (pmg) was found to form very strong complexes with DNA. This eventually helped us to monitor the number of phosphate groups in modified oligonucleotides [22]. In view of the importance of noncovalent binding interactions involving the guanidinium group and acidic moieties [23], we decided to shift our attention toward MALDI detection of sulfateguanidinium interactions. Our goal was to develop an analytical tool that could reveal in a single experiment the degrees of polymerization (molecular weight) and sulfation (number of sulfate groups) of the analyte. Such a method would allow rapid and confident char- acterizations of sulfated oligosaccharides that are usually hampered by uncertainty.

The work presented here describes a very efficient tool for matrix-assisted laser desorption/ionization of polyanionic oligosaccharides using pyrenemethylguanidine. The method was used to analyze various highly sulfated chondroitin and carrageenan oligosaccharides as well as the sodium salt of sucrose octasulfate. Unique pmg-complexed ladders of peaks have been observed for all of the di- to decasaccharides carrying from two to nine sulfate groups analyzed.

\section{Experimental}

\section{Materials}

All chemicals were purchased from commercial sources and used without further purification. Sucrose octasulfate sodium salt was a gift from EUTICALS (Milano, Italy). Chondroitin sulfates were synthesized according to known procedures $[4,24]$. Carrageenans were prepared from various algae, as previously reported, and their structures were previously confirmed by spectroscopic analysis [25-27]. In somes cases (see, for example, spectra S16 and S19 in the supplementary material, which can be found in the electronic version of this article) the analysis of carrageenans gave rise to the presence of either minor peaks or main peaks present as a doublet. These results, usually observed in both ionization modes for a given compound, are not the consequence of glycosidic cleavage fragmentations but rather reflect the purity of the analytes obtained after enzymatic digestion and purification of complex hybrid carrageenans. These impurities are attributed to the presence of the corresponding anhydro analogues. This further demonstrates the value of this method in the assessment of the purity of compounds.

\section{Mass Spectrometry}

The MALDI mass spectra were recorded on a Voyager DE (PerSeptive Biosystems, Framingham, MA, USA) equipped with a 337-nm nitrogen laser. All mass spectra were acquired in linear mode with an acceleration voltage of $20 \mathrm{kV}$; guide wire, $0.05 \%$ of accelerating voltage; $94 \%$ of accelerating voltage; delay extraction time, $500 \mathrm{~ns}$. Typically 50 laser shots were averaged for each spectrum. Sulfated oligosaccharide solutions $(0.1$ $\mathrm{mM}$ ) were prepared in deionized water. Pyrenemethylguanidine hydrochloride [28] was dissolved in deionized water and $\mathrm{MeOH}$ (1:1; final concentration $10 \mathrm{mM})$. The matrix solution was prepared daily by dissolving p-nitroaniline in EtOH (final concentration $0.1 \mathrm{M}$ ). For sensitivity determination, a series of dilutions of chondroitin CSC hexa (for structures see the supplementary material) was done (final concentrations 50, 5, and 0.5 $\mu \mathrm{M})$ in the presence of 30-300 equivalents of pmg. Samples were prepared by mixing $5 \mu \mathrm{L}$ of the oligosaccharide solution $\left(0.1 \mathrm{mM}\right.$ in $\left.\mathrm{H}_{2} \mathrm{O}\right), 2 \mu \mathrm{L}$ of pyrenemeth- 
ylguanidine hydrochloride solution (10 mM in $\mathrm{MeOH} /$ $\left.\mathrm{H}_{2} \mathrm{O} 1: 1\right)$, and $3 \mu \mathrm{L}$ of deionized water. This mixture $(1 \mu \mathrm{L})$ was deposited on the MALDI plate and directly mixed on the plate with an ethanolic solution of $p$ nitroaniline $(1 \mu \mathrm{L}, 0.1 \mathrm{M})$ for $15 \mathrm{~s}$. The resulting mixture was allowed to dry for $10 \mathrm{~min}$ at room temperature before being applied to MALDI-MS analysis.

\section{Mixture Analysis}

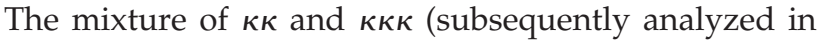
Figure 4) was obtained by mixing $2.5 \mu \mathrm{L}$ of $\kappa \kappa(0.1 \mathrm{mM}$ in $\left.\mathrm{H}_{2} \mathrm{O}\right)$ and $\kappa \kappa \kappa\left(0.1 \mathrm{mM}\right.$ in $\left.\mathrm{H}_{2} \mathrm{O}\right), 2 \mu \mathrm{L}$ of pyrenemethylguanidine hydrochloride solution $(10 \mathrm{mM}$ in $\left.\mathrm{MeOH} / \mathrm{H}_{2} \mathrm{O}, 1: 1\right)$, and $3 \mu \mathrm{L}$ of deionized water.

\section{Carrageenan Software Interface}

The Carrageenan analyzer is a simple, downloadable, Excel spreadsheet designed as a software application to determine the possible composition of carrageenans from data obtained by MALDI-MS experiments (see Supplementary Material available in the online version of this article). Although this version analyses only dito decasaccharides that are composed of $\kappa^{-}, \iota^{-}, \mu-$, or $\lambda$-carrabiose units, this program could be easily modified by the user to extend the degrees of polymerization or to add other carrabiose units. This software tool was developed using the "IF" function of Microsoft Excel. First, all possible oligocarrageenan combinations up to decasaccharides were generated and their theoretical fully complexed molecular masses were calculated. The conditions for the "IF" function were next defined with a margin of mass error (in this version $\pm 0.14 \%$ of theoretical mass, except for decasaccharide units where a margin of mass error of $\pm 0.13 \%$ was set). These tolerances allow the length of the saccharide based on their fully complexed molecular weight and the number of sulfate groups to be uniquely assigned. Indeed, the minimum difference within a series of di-, tetra-, hexa-, or decasaccharide is $18 \mathrm{Da}$. Further, there is a minimum difference in mass of 64.9 Da between di- and tetrasaccharides, 46.8 Da between tetra- and hexasaccharides, 28.8 Da between hexa- and octasaccharides, and 10.8 Da between octa- and decasaccharides. These molecular mass differences are always superior to the defined margin of mass error. The masses of the fragments ions identified in our study always matched those generated by calculation within the accuracy defined in the software. Therefore, the length of the oligosaccharide and the number of sulfate groups are accurately assigned for any given oligosaccharide up to decasaccharides. Upon counting peaks observed in a MALDI experiment, between which the differences of mass must be $353 \mathrm{Da}$, one should (1) enter this number in the Peak cell and (2) enter the highest mass value in the Mass cell. The application will then (3) generate possible compositions in the composition cell. Matching composition should be generated by both positive- and negative- ionization mode analyzers. In some cases, fully desulfated ions (generally in the positive-ionization mode) or fully complexed ions (generally in the negative-ionization mode) might be observed as small peaks. It is therefore important to make sure that spectra obtained in both ionization modes match the general rules developed in the article. Given the margin of mass error defined, the probability of a false hit is greatly reduced by this cross-examination.

\section{Results and Discussion}

\section{Analysis of Chondroitin Sulfates}

In the process of selecting the best conditions to obtain excellent MALDI mass spectra we selected the nonacidic p-nitroaniline [29] as a matrix in the presence of 40 equivalents of pmg. This matrix offered best shot-toshot reproducibility and signal stability in the analysis. Typically, 50 laser shots were averaged for each spectrum without noticing any signal deterioration.

We first focused on the analysis of chondroitin sulfates (CS). Chondroitin sulfates are linear copolymers made of dimeric units composed of D-glucuronic acid (GlcA) and 2-acetamido-2-deoxy-D-galactose (GalNAc) arranged in the sequence $[\rightarrow 4)-\beta$-D-GlcA- $(1 \rightarrow 3)-\beta$-D-GalNAc- $(1 \rightarrow]_{n}$, and contain on average one sulfate group per disaccharide unit, but other types with sulfate(s) at various positions are also known (Figure 1). MALDI-TOF analysis was performed on synthetic samples of chondroitin
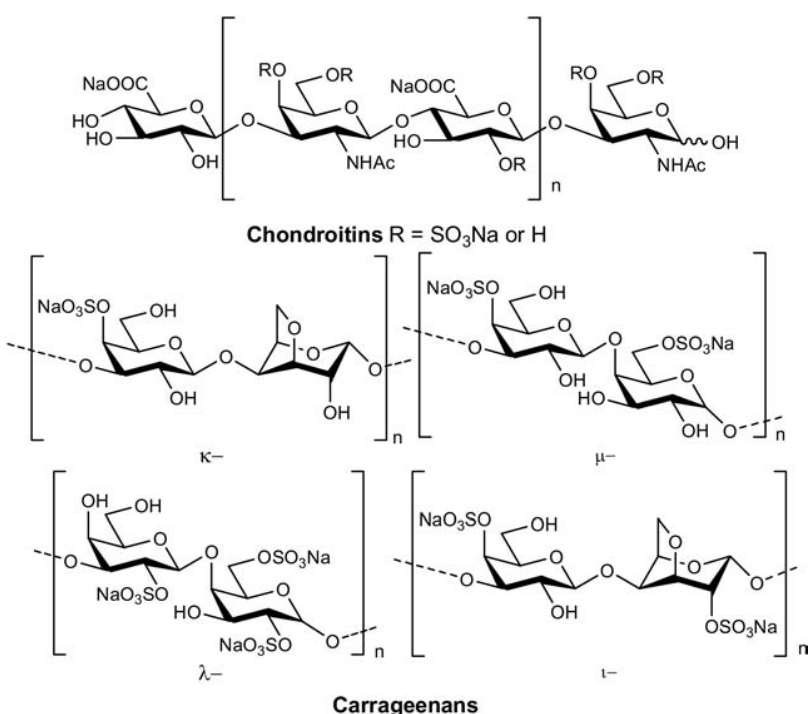

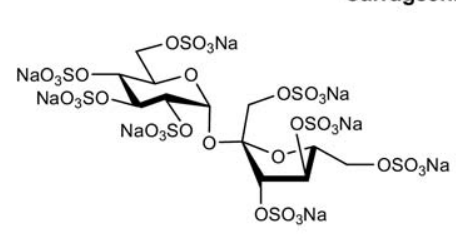

Sucrose Octasulfate

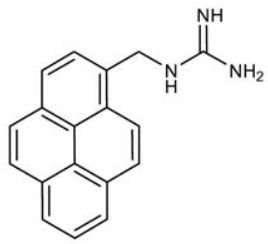

pmg
Figure 1. Structures of sulfated oligosaccharides analyzed using pyrenemethylguanidine (pmg). 
sulfates with various sulfation patterns in the negativeand positive-ionization modes. Although no signal could be observed without pmg, the addition of 40 equivalents $\left(\mathrm{MW}_{\mathrm{pmg}}=273 \mathrm{~g} \mathrm{~mol}^{-1}\right)$ of the latter gave a ladder of peaks dependent on the number of sulfate groups in both ionization modes. An illustrative example is given in Figure 2 with the tetrasaccharide tetrasulfated CS-D tetra. In the positive mode, a ladder of five peaks is obtained corresponding to the complexation of $5 \mathrm{pmg}$ with the oligosaccharide. The fully complexed ion is easily detected at $m / z 2476.7$, whereas the calculated value is 2476.6. In the negative mode, four well-distinguished peaks can be observed, with the highest $\mathrm{m} / \mathrm{z}$ value at 1927.5 corresponding to a complexation of CS-D tetra with 3 pmg molecules (calcd. 1928.4). The mass differences between the observed peaks in both ionization modes correspond to a $\mathrm{SO}_{3}-$ pmg cleavage with an $\mathrm{H}$ substituting the $\mathrm{SO}_{3}$-pmg pair (net loss of 353 mass units). This specific $\mathrm{SO}_{3}-$ pmg loss associated with the highest $\mathrm{m} / \mathrm{z}$ value is very useful because it does not create any ambiguity in the data interpretation. The molecular weight of the fully protonated analyte is therefore deduced from these spectra: MW = 1109.7 Da (calcd. 1110.1). Changing the laser energy did not affect the number of observed fragments and influences only their respective intensities. For all CS analyzed, mass spectra followed the general formula of $n$ peaks in the negative mode and $[n+1]$ peaks in the positive mode, where $n$ is the degree of sulfation of the analytes. In the positive mode these results indicate that all $\mathrm{SO}_{3}$ groups are bound to a pmg molecule as is one of the carboxylate groups. This result seems to indicate that the sulfate-pmg complex is stronger than the carboxylate-pmg one. By contrast, the negative-ion analysis indicates that one of the sulfate groups is always staying free. The highest $\mathrm{m} / \mathrm{z}$ values were found to follow the general formula $m / z=\left[\mathrm{M}_{\mathrm{OS}}+(n+1) \times\right.$ $\left.\mathrm{M}_{\mathrm{pmg}}+\mathrm{H}\right]^{+}$in the positive mode and $m / z=\left[\mathrm{M}_{\mathrm{OS}}+\right.$ $\left.(n-1) \times \mathrm{M}_{\mathrm{pmg}}-\mathrm{H}\right]^{-}$in the negative mode, where $\mathrm{M}_{\mathrm{OS}}$ and $\mathrm{M}_{\mathrm{pmg}}$ refer to the exact mass of the fully protonated oligosaccharide and the guanidine derivative, respectively. Additionally, the peaks with the lowest $\mathrm{m} / \mathrm{z}$ values correspond to the fully desulfated ion in the positive mode and to a monosulfated ion in the negative mode. No sodium adducts and no glycosidic cleavage fragments were detected whatsoever. This method is equally useful to analyze less sulfated CS as well as phosphorylated CS (see the supplementary material). Sensitivity was assessed and reproducible analyte signals could be obtained in both ionization modes with 10 pmol of sample material per target spot. A detection limit of 1 pmol can be achieved with 300 equivalents of pmg in the negative mode without affecting the quality of the spectra, thus attaining sensitivity within a biologically relevant range of analyte concentrations. Because the molecular ratio of analyte versus pyrenemethylgua-
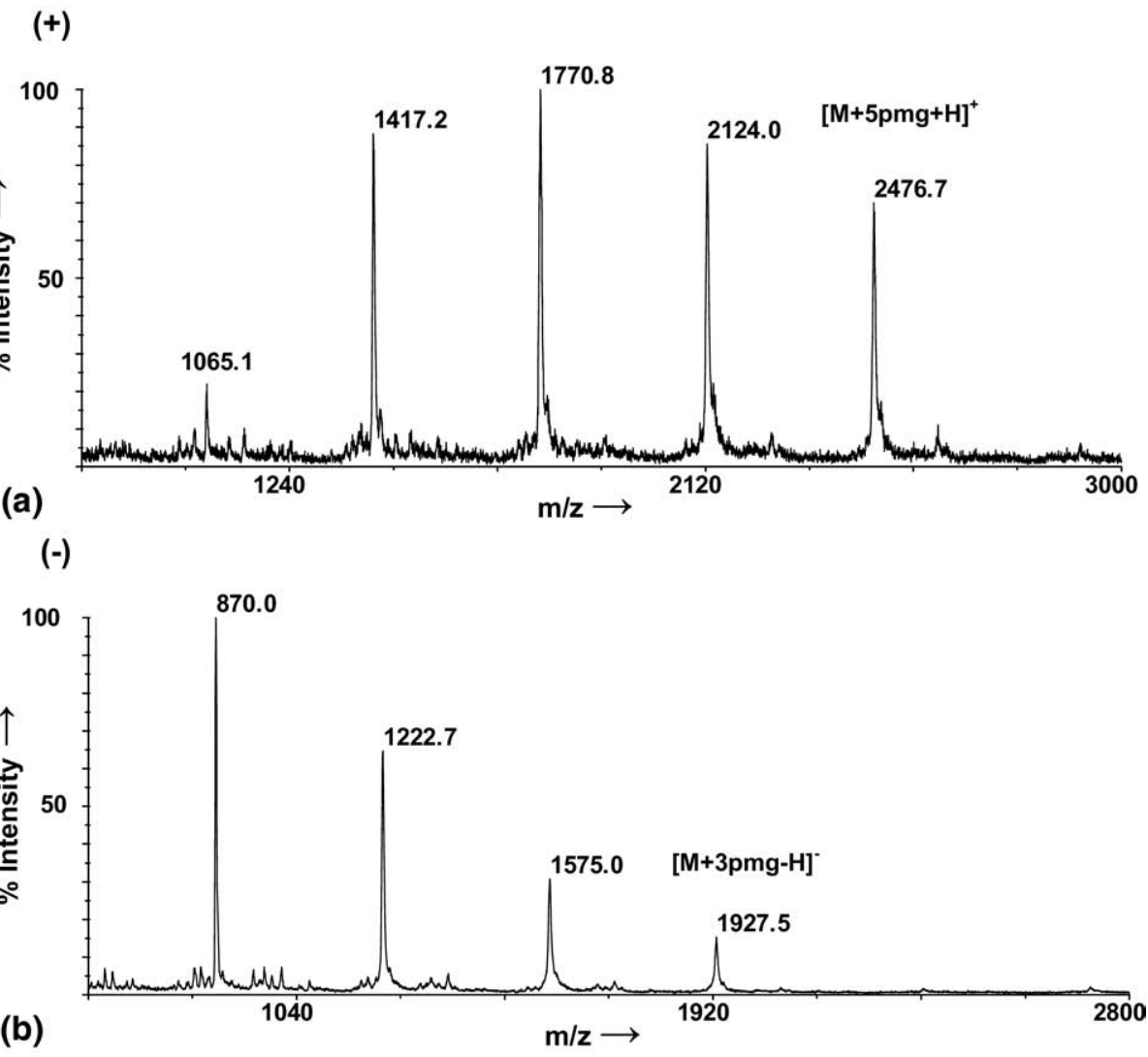

Figure 2. Positive-ion (a) and negative-ion (b) MALDI mass spectra of CS-D tetra with pmg. 
nidine is an important factor for the observation of the fully complexed oligosaccharide form, an easy way to analyze samples whose amounts are unknown is to increase the concentration of the pmg solution until no intensity change of the maximum $\mathrm{m} / \mathrm{z}$ ion is observed.

\section{Analysis of Carrageenans}

We next shifted our attention toward the carrageenans, another source of highly sulfated polysaccharides. Carrageenans are composed of galactose and 3,6-anhydrogalactose, linked by alternating $\alpha 1,3$ and $\beta 1,4$ glycosidic linkages. The disaccharide residues may variously be sulfated, and differences in degree of sulfation and occurrence of the 3,6-anhydrogalactose residues give rise to the various forms of carrageenans. Of these forms, $\lambda$ - (D2S6S-G2S), $\kappa^{-}$(DA-G4S), $\mu$ - (D6SG4S), and $\iota$ (DA2S-G4S) carrageenans are probably the most important (Figure 1) and the heterogeneity of the carrageenans renders them particularly difficult to characterize. Like chondroitins no usable spectra could be obtained without addition of pmg. However, applying our method to various carrageenans differing in both length and sulfation patterns, we were again pleased to observe specific ladders of peaks that directly relate the number of sulfate groups depending on the number of observed fragments ( $\Delta=353$ mass units). Because carrageenans do not bear carboxylate functions, mass spectra followed in both ionization modes the general formula of $n$ peaks for $n$ sulfate groups. As shown in Figure 3 with the decasaccharide heptasulfated hybrid sequence $\kappa \kappa \mu \mu \kappa$, the highest $m / z$ values (4330.2 in the positive mode and 3781.6 in the negative mode) follow the same general formula found for the chondroitins. However, because of the absence of a carboxylic function, a difference with the chondroitins is observed with the lowest $m / z$ values, which correspond in both ionization modes to a monosulfated ion. Because a series of seven peaks ( $\Delta=353$ mass units) are observed in both ionization modes, we confirm that the molecule is heptasulfated with a molecular weight of $2144.2 \mathrm{Da}$ (calcd. 2144.2). The high efficiency and reproducibility of our analytical method prompted us to develop an algorithm for the rapid and automatic interpretation of the mass spectra obtained in both positive- and negativeionization modes, therefore facilitating the process of deriving sequence information (see the supplementary material). These basic software applications were particularly useful for oligosaccharide mixtures analysis. As exemplified in Figure 4 a mixture of $\kappa \kappa$ and $\kappa \kappa \kappa$ carrageenans led to two series of peaks differing in intensities in both ionization modes. These two series can be identified by the 353 mass-unit difference observed between each peak. Summing the number of peaks (2 and 3, respectively) and entering the $\mathrm{m} / \mathrm{z}$

a) $(+)$

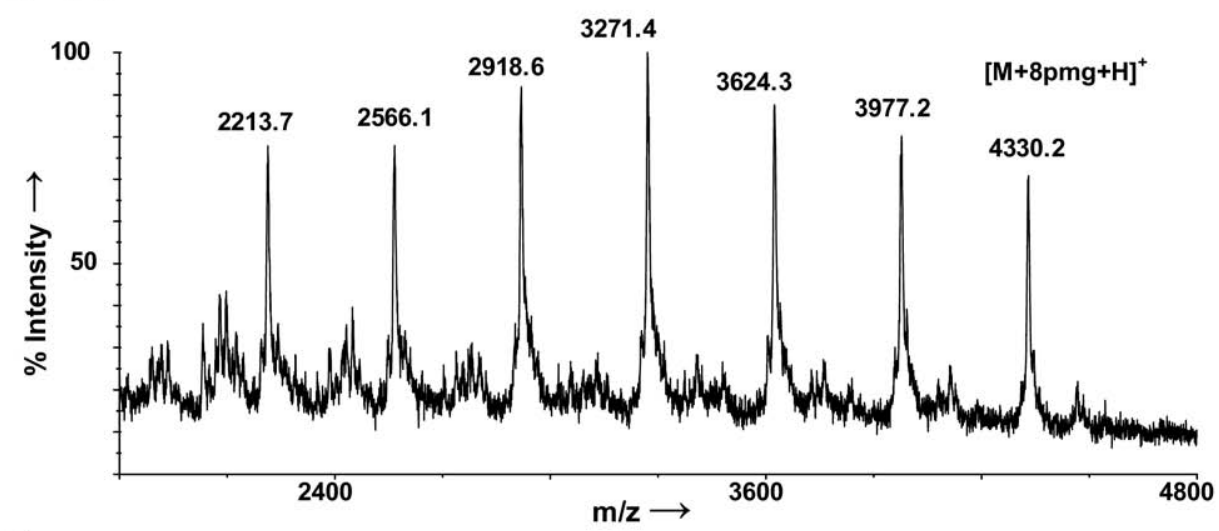

b) (-)

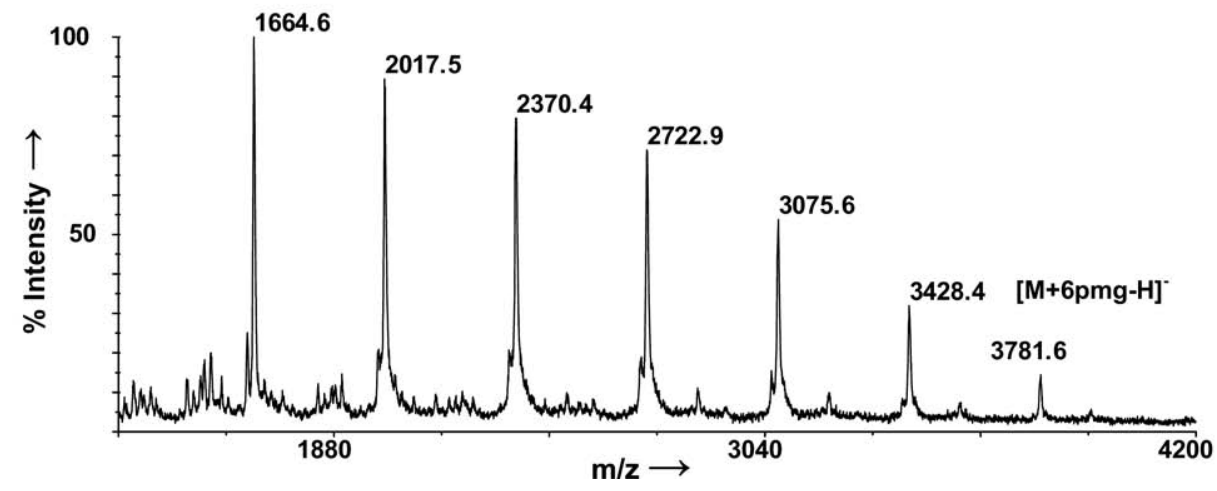

Figure 3. Positive-ion (a) and negative-ion (b) MALDI mass spectra of $\kappa \kappa \mu \mu \kappa$ with pmg. 


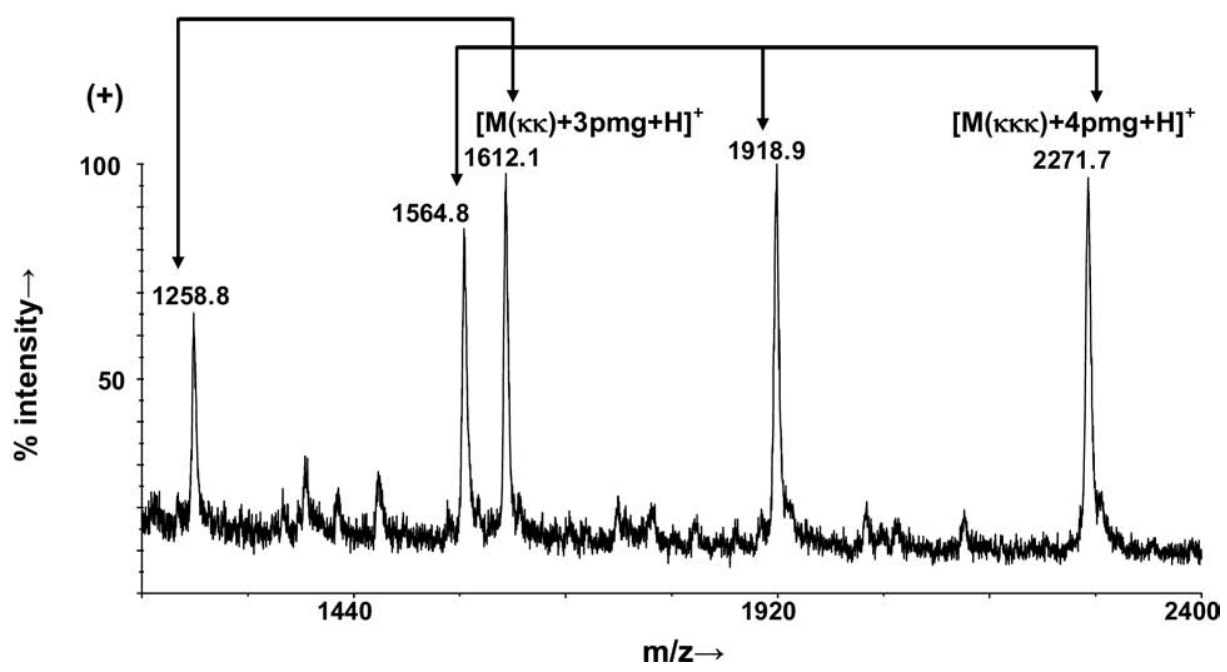

Figure 4. Positive-ion MALDI mass spectrum of a mixture of $\kappa \kappa$ and $\kappa \kappa \kappa$ with pmg.

values of the fully sulfated ions into our applications $(\mathrm{m} / \mathrm{z} 1612.1$ and 2271.7, respectively, in the positiveionization mode and 1062.5 and 1720.9 in the negative-ionization mode, respectively) generate in both ionization modes the composition of the corresponding oligosaccharides: $\kappa \kappa$ and $к \kappa \kappa$. The perfect match obtained for both ionization modes allows a confident structural characterization of unknown carrageenans. When these compounds were analyzed individually their respective spectra gave peaks at $\mathrm{m} / \mathrm{z}$ 1258.7 and 1612.1 for $\kappa \kappa$ and 1564.7, 1918.9, and 2271.6 for $\kappa \kappa \kappa$. These results demonstrate that this pmg-complexing method can be successfully used for the characterization of depolymerized mixtures without affecting mass spectra analysis. It should be noted that specific arrangements could not be determined without digestion experiments and that mixtures of carrageenans having the same backbone but different numbers of sulfates (e.g., $к \kappa \kappa$ and $\kappa \iota \kappa)$ are indistinguishable in mixture analysis, although they can be separated by chromatography.

\section{Sucrose Octasulfate Analysis}

Finally, our method was evaluated on the sodium salt of the disaccharide sucrose octasulfate (SOS, Figure 1). This compound is the active ingredient of the antiulcer drug sucralfate and was chosen for its exceedingly high degree of sulfation. The mass spectra obtained in both ionization modes were found to follow the rules concluded from the carrageenans analysis. A series of eight peaks $(\Delta=353$ mass units) are observed in both ionization modes, but it should be noted that the positive mode gave better sensitivity, in that 50 equivalents of pmg were needed to obtain the fully complexed form in the negative mode (Figure 5). Nevertheless, neither sodium exchange nor glycosidic cleavage was observed, further demonstrating the scope of our method.

\section{Conclusions}

A new powerful method for the MALDI-MS analysis of polysulfated oligosaccharides using pyrenemethylgua-

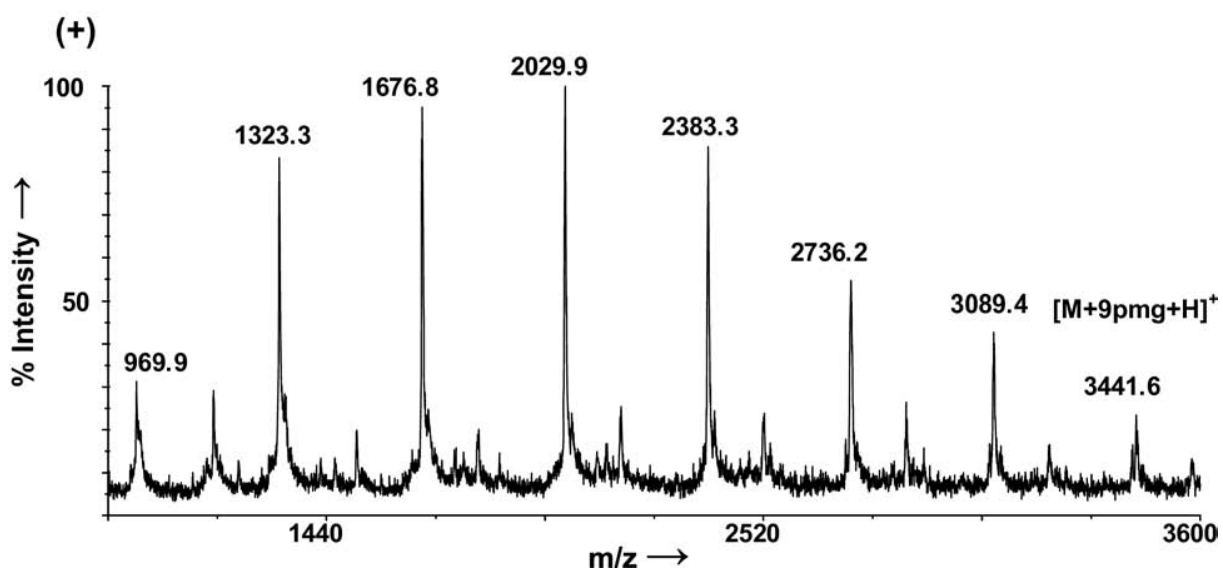

Figure 5. Positive-ion MALDI mass spectrum of SOS with pmg. 
nidine as an effective derivatizing agent and ionization enhancer was introduced. Although fragmentation is generally considered a major inconvenience, the generation of specific fragments resulting from $\mathrm{SO}_{3}-\mathrm{pmg}$ cleavages, with an $\mathrm{H}$ substituting the $\mathrm{SO}_{3}-$ pmg pairs, has a number of distinctive advantages. It allows the detection and the molecular weight determination of highly sulfated oligosaccharides through the formation of complexes with pmg. Specific fragmentations permit the unambiguous determination of the sulfation degree of the molecule. The analytical utility of the complex formation is demonstrated by the molecular mass determination of compounds from 500 to $5000 \mathrm{Da}$ at the picomole level with accuracy of $\pm 0.1 \%$ and the absence of alkali cation adduct. Adding pmg is very suitable for the direct analysis of purified polysulfated oligosaccharides up to decasaccharides, as well as oligosaccharide mixtures. Finally, through the development of our software, we provided a practical methodology for the analysis of carrageenans of unknown structures as a list of all possible structures is quickly generated from information obtained in the mass spectra.

Future studies will be directed toward the application of this method to more complex mixtures as well as other class of GAG-derived oligosaccharides such as heparin oligosaccharides. Monitoring enzymatic depolymerization reactions using this method will also be evaluated.

\section{Acknowledgments}

We gratefully acknowledge Université de Montpellier 2 for financial support and Euticals SA (Italy) for a generous gift of SOS. KO thanks "l'Association pour la Recherche contre le Cancer" (ARC) for a doctoral fellowship. We thank the reviewers for their comments.

\section{References}

1. Esko, J. D.; Selleck, S. B. Order Out of Chaos: Assembly of Ligand Binding Sites in Heparan Sulfate. Annu. Rev. Biochem. 2002, 71, 435-471.

2. Linhardt, R. J.; Toida, T. Role of Glycosaminoglycans in Cellular Communication. Acc. Chem. Res. 2004, 37, 431-438.

3. Capila, I.; Linhardt, R. J. Heparin-Protein Interactions. Angew. Chem. Int. Ed. Engl. 2002, 41, 391-412.

4. Lopin, C.; Jacquinet, J. C. From Polymer to Size-defined Oligomers: An Expeditious Route for the Preparation of Chondroitin Oligosaccharides. Angew. Chem. Int. Ed. Engl. 2006, 45, 2574-2578.

5. van de Velde, F.; Peppelman, H. A.; Rollema, H. S.; Tromp, R. H. On the Structure of kappa/iota-Hybrid Carrageenans. Carbohydr. Res. 2001, 331, 271-283.

6. Menard, R.; de Ruffray, P.; Fritig, B.; Yvin, J. C.; Kauffmann, S. Defense and Resistance-inducing Activities in Tobacco of the Sulfated beta-1,3 Glucan PS3 and Its Synergistic Activities with the Unsulfated Molecule. Plant Cell Physiol. 2005, 46, 1964-1972.

7. McReynolds, K. D.; Gervay-Hague, J. Chemotherapeutic Interventions Targeting HIV Interactions with Host-associated Carbohydrates. Chem. Rev. 2007, 107, 1533-1552.
8. van de Velde, F.; Knutsen, S. H.; Usov, A. I.; Rollema, H. S.; Cerezo, A. S H-1 and C-13 High Resolution NMR Spectroscopy of Carrageenans: Application in Research and Industry. Trends Food Sci. Technol. 2002, 13, 73-92.

9. Chi, L.; Amster, J.; Linhardt, R. J. Mass Spectrometry for the Analysis of Highly Charged Sulfated Carbohydrates. Curr. Anal. Chem. 2005, 1, 223-240.

10. Chai, W. G.; Luo, J.; Lim, C. K.; Lawson, A. M. Characterization of Heparin Oligosaccharide Mixtures as Ammonium Salts Using Electrospray Mass Spectrometry. Anal. Chem. 1998, 70, 2060-2066.

11. Gunay, N. S.; Tadano-Aritomi, K.; Toida, T.; Ishizuka, I.; Linhardt, R. J. Evaluation of Counterions for Electrospray Ionization Mass Spectral Sulfated Analysis of a Highly Sulfated Carbohydrate, Sucrose Octasulfate. Anal. Chem. 2003, 75, 3226-3231.

12. Siegel, M. M.; Tabei, K.; Kagan, M. Z.; Vlahov, I. R.; Hileman, R. E. Linhardt, R. J. Polysulfated Carbohydrates Analyzed as Ion-paired Complexes with Basic Peptides and Proteins Using Electrospray Negative Ionization Mass Spectrometry. J. Mass Spectrom. 1997, 32, 760-772.

13. Zaia, J. Mass Spectrometry of Oligosaccharides. Mass Spectrom. Rev. 2004, 23, 161-227.

14. Zaia, J. Li, X. Q.; Chan, S. Y.; Costello, C. E. Tandem Mass Spectrometric Strategies for Determination of Sulfation Positions and Uronic Acid Epimerization in Chondroitin Sulfate Oligosaccharides. J. Am. Soc. Mass Spectrom. 2003, 14, 1270-1281.

15. Juhasz, P.; Biemann, K. Mass-Spectrometric Molecular-Weight Determination of Highly Acidic Compounds of Biological Significance via Their Complexes with Basic Polypeptides. Proc. Natl. Acad. Sci. U. S. A. 1994, 91, 4333-4337.

16. Juhasz, P.; Biemann, K. Utility of Noncovalent Complexes in the MatrixAssisted Laser-Desorption Ionization Mass-Spectrometry of HeparinDerived Oligosaccharides. Carbohydr. Res. 1995, 270, 131-147.

17. Venkataraman, G.; Shriver, Z.; Raman, R.; Sasisekharan, R. Sequencing Complex Polysaccharides. Science 1999, 286, 537-542.

18. Laremore, T. N.; Murugesan, S.; Park, T. J.; Avci, F. Y.; Zagorevski, D. V.; Linhardt, R. J. Matrix-Assisted Laser Desorption/Ionization Mass Spectrometric Analysis of Uncomplexed Highly Sulfated Oligosaccharides Using Ionic Liquid Matrices. Anal. Chem. 2006, 78, 1774-1779.

19. Laremore, T. N.; Zhang, F. M.; Linhardt, R. J. Ionic Liquid Matrix for Direct UV-MALDI-TOF-MS Analysis of Dermatan Sulfate and Chondroitin Sulfate Oligosaccharides. Anal. Chem. 2007, 79, 1604-1610.

20. Tissot, B.; Gasiunas, N.; Powell, A. K.; Ahmed, Y.; Zhi, Z. L.; Haslam, S. M.; Morris, H. R.; Turnbull, J. E.; Gallagher, J. T.; Dell, A. Towards GAG Glycomics: Analysis of Highly Sulfated Heparins by MALDI-TOF Mass Spectrometry. Glycobiology 2007, 17, 972-982.

21. Laremore, T. N.; Linhardt, R. J. Improved Matrix-Assisted Laser Desorption/Ionization Mass Spectrometric Detection of Glycosaminoglycan Disaccharides as Cesium Salts. Rapid Commun. Mass Spectrom. 2007, 21, 1315-1320.

22. Ohara, K.; Smietana, M.; Vasseur, J. J. Characterization of Specific Noncovalent Complexes between Guanidinium Derivatives and Singlestranded DNA by MALDI. J. Am. Soc. Mass Spectrom. 2006, 17, 283-291.

23. Schug, K. A.; Lindner, W. Noncovalent Binding between Guanidinium and Anionic Groups: Focus on Biological- and Synthetic-based Arginine/ Guanidinium Interactions with Phosph[on]ate and Sulf[on]ate Residues. Chem. Rev. 2005, 105, 67-113.

24. Karst, N.; Jacquinet, J. C. Stereocontrolled Total Syntheses of Shark Cartilage Chondroitin Sulfate D-related Tetra- and Hexasaccharide Methyl Glycosides. Eur. J. Org. Chem. 2002, 5, 815-825.

25. Guibet, M.; Boulenguer, P.; Mazoyer, J.; Kervarec, N.; Antonopoulos, A. Lafosse, M.; Helbert, W. Composition and Distribution of Carrabiose Moieties in Hybrid kappa-/iota-Carrageenans Using Carrageenases. Biomacromolecules 2008, 9, 408-415.

26. Guibet, M.; Colin, S.; Barbeyron, T.; Genicot, S.; Kloareg, B.; Michel, G. Helbert, W. Degradation of lambda-Carrageenan by Pseudoalteromonas carrageenovora lambda-Carrageenase: A New Family of Glycoside Hydrolases Unrelated to kappa- and iota-Carrageenases. Biochem. J. 2007, 404, 105-114

27. Guibet, M.; Kervarec, N.; Genicot, S.; Chevolot, Y.; Helbert, W. Complete Assignment of H-1 and C-13 NMR Spectra of Gigartina skottsbergii lambda-Carrageenan Using Carrabiose Oligosaccharides Prepared by Enzymatic Hydrolysis. Carbohydr. Res. 2006, 341, 1859-1869.

28. Ohara, K.; Smietana, M.; Restouin, A.; Mollard, S.; Borg, J. P.; Collette, Y.; Vasseur, J. J. Amine-Guanidine Switch: A Promising Approach to Improve DNA Binding and Antiproliferative Activities. J. Med. Chem. 2007, 50, 6465-6475.

29. Salih, B.; Zenobi, R. MALDI Mass Spectrometry of Dye-Peptide and Dye-Protein Complexes. Anal. Chem. 1998, 70, 1536-1543. 